OPEN ACCESS

Edited by:

Hyang Burm Lee,

Chonnam National University,

South Korea

Reviewed by:

Sanjaya Gyawali,

Washington State University,

United States

Hyunkyu Sang,

Chonnam National University,

South Korea

*Correspondence:

W. G. Dilantha Fernando

Dilantha.Fernando@umanitoba.ca

tORCID:

Zhongwei Zou

orcid.org/0000-0002-8955-1153

Specialty section:

This article was submitted to

Fungi and Their Interactions,

a section of the journal

Frontiers in Microbiology

Received: 27 March 2020

Accepted: 24 July 2020

Published: 11 August 2020

Citation:

Zou Z, Liu F, Selin C and Fernando WGD (2020) Generation and Characterization of a Virulent Leptosphaeria maculans Isolate Carrying a Mutated AvrLm7 Gene Using the CRISPR/Cas9 System.

Front. Microbiol. 11:1969. doi: 10.3389/fmicb.2020.01969

\section{Generation and Characterization of a Virulent Leptosphaeria maculans Isolate Carrying a Mutated AvrLm7 Gene Using the CRISPR/Cas9 System}

\author{
Zhongwei Zou', Fei Liu, Carrie Selin and W. G. Dilantha Fernando* \\ Department of Plant Science, University of Manitoba, Winnipeg, MB, Canada
}

Blackleg, caused by the fungal pathogen Leptosphaeria maculans, is the most important disease affecting canola (Brassica napus) crops worldwide. We employed the clustered regularly interspaced short palindromic repeat (CRISPR)/CRISPR-associated (Cas) system to generate the mutant isolate umavr7 from a point mutation of the AvrLm7 coding region in a L. maculans isolate (UMAvr7). Reverse transcription PCR and transcriptome data confirmed that the AvrLm7 gene was knocked out in the mutant isolate. Pathogenicity tests indicated that umavr7 can cause large lesions on a set of Brassica differential genotypes that express different resistance $(R)$ genes. Comparative pathogenicity tests between UMAvr7 (wild type) and umavr7 on the corresponding B. napus genotype 01-23-2-1 (with R/m7) showed that umavr7 is a mutant isolate, producing large gray/green lesions on cotyledons. The pathogenicity of the mutant isolate was shifted from avirulent to virulent on the B. napus RIm7 genotype. Therefore, this mutant is virulence on the identified resistant genes to blackleg disease in $B$. napus genotypes. Superoxide accumulated differently in cotyledons in response to infection with UMAvr7 and umavr7, especially in resistant B. napus genotype 01-23-2-1. Resistance/susceptibility was further evaluated on 123 B. napus genotypes with the mutant isolate, umavr7. Only 6 of the 123 genotypes showed resistance to umavr7. The identification of these six resistant $B$. napus genotypes will lead to further studies on the development of blackleg disease resistance through breeding and the identification of novel $R$ genes.

Keywords: Leptosphaeria maculans, canola, gene editing, breeding for disease resistance, RIm7, AvrLm7, CRISPR/Cas9, Brassica napus

\section{INTRODUCTION}

Blackleg, caused by the fungal pathogen Leptosphaeria maculans, is the most important disease affecting canola (Brassica napus) crops worldwide (Fitt et al., 2006). Since the 1990's, blackleg has been identified as a major disease in Canadian canola and has resulted in significant yield losses (Gugel and Petrie, 1992). Blackleg disease severity and the extent of canola yield loss vary with geographic distribution, climate, cultivar and crop rotation. In the last four decades, blackleg has 
been mainly controlled by the use of resistant cultivars and crop rotation (Fernando et al., 2016). However, L. maculans is considered a highly virulent and globally invasive species (Fitt et al., 2006; Zhang and Fernando, 2018). Previous studies have indicated that a gene-for-gene relationship exists between each $R$ gene in canola and its corresponding avirulence gene (Avr) in the L. maculans isolate (Ansan-Melayah et al., 1998; Balesdent et al., 2002). $R$ gene-mediated resistance, based on this relationship, is used so frequently that many blackleg disease management strategies have been developed, and cultivars have been bred for resistance to the fungal populations that harbor the majority of the corresponding avirulence alleles (Daverdin et al., 2012).

To date, a total of 16 Avr genes have been identified in L. maculans, including AvrLm1-11, AvrLepR1-LepR3, and AvrLmS (Gout et al., 2006; Hayward et al., 2012; Van de Wouw et al., 2014; Ghanbarnia et al., 2015; Plissonneau et al., 2016). Among these, nine avirulence genes have been cloned: AvrLm1 (Gout et al., 2006), AvrLm3 (Plissonneau et al., 2016), AvrLm6 (Fudal et al., 2007), AvrLm5(J1) (Van de Wouw et al., 2014), AvrLm2 (Ghanbarnia et al., 2015), AvrLm4-7 (Parlange et al., 2009), AvrLm11 (Balesdent et al., 2013), AvrLm9 (cosegregating with AvrLm5) (Ghanbarnia et al., 2018), and AvrLm10 (Petit-Houdenot et al., 2019). A recent study indicated that AvrLmJ1 maps to the same position as AvrLm5; isolates carrying the AvrLmJ1 locus confer avirulence toward Brassica juncea genotypes with the Rlm5 gene, demonstrating that AvrLmJ1 is AvrLm5 (Plissonneau et al., 2018). Correspondingly, various major resistance genes in Brassica species have been identified, including Rlm1-11, LepR1-LepR4, RlmS, BLMR1, and BLMR2 (Chèvre et al., 1997; Rimmer, 2006; Van de Wouw et al., 2009; Eber et al., 2011; Long et al., 2011). Among these, Rlm5 and Rlm6 are found in Brassica juncea (AABB genome) (Chèvre et al., 1997; Balesdent et al., 2002; Van de Wouw et al., 2014). One resistance gene, within two alleles of LepR3/Rlm2, which interacts with AvrLm1 and AvrLm2, respectively, has been cloned (Larkan et al., 2013 and 2015).

In the past decade, the predominant tools employed to achieve site-directed double strand breaks are ZFNs (zinc-finger nucleases) and TALENS (transcription activator-like effector nucleases), both of which have provided invaluable assistance in targeted gene mutation in a diverse range of organisms. More recently, the clustered regularly interspaced short palindromic repeats (CRISPR)-Cas (CRISPR-associated) protein system has gained much attention and has been widely used for editing the genomes of animals, plants and fungi. In comparison to ZFNs and TALENS, CRISPR/Cas9 is easier to manipulate, as it employs a RNA-guide nuclease, Cas9, to induce double-strand breaks for mutation and can be recycled to edit different targets. CRISPR/Cas9 has been used for targeted mutagenesis in various plant species, including Arabidopsis (Jiang et al., 2013), Nicotiana tabacum (Li et al., 2013), potato (Shan et al., 2013), rice (Xie and Yang, 2013), tomato (Brooks et al., 2014), and soybean (Cai et al., 2015). The CRISPR system has also been used extensively in filamentous fungi to decode fungal pathogenesis (Deng et al., 2017), and Takayuki et al. (2015) developed a modified tailormade CRISPR/Cas system to increase targeted gene replacement efficiency in the rice blast fungus. Comparative analyses of secreted proteins in plant pathogenic smut fungi and related basidiomycetes have been conducted using the CRISPR/Cas9 toolkit for simultaneous disruption of multiple genes (Schuster et al., 2018). Very recently, Idnurm et al. (2017) optimized a CRISPR/Cas9 system to disrupt the hos1 gene, encoding a predicted osmosensing histidine kinase, in different $L$. maculans strains. The L. maculans strains with the mutated hos 1 gene showed reduced growth under high salt conditions but were still able to cause lesions on canola plants.

From a 6-year blackleg disease survey, examining the dynamic avirulence allele profiles of L. maculans isolates in Manitoba, Canada, it was evident that the fungal populations carry a diverse number of Avr genes. A total of 180 Avr gene races were identified from 964 isolates, with three major races observed: AvrLm-2-45-6-7, AvrLm2-4-5-6-7-S, and AvrLm-1-4-5-6-7-11-(S). Very few isolates carried a single Avr gene conferring virulence to most commercial canola cultivars (Fernando et al., 2018). However, a L. maculans isolate (DS103) with few Avr genes, termed UMAvr7, was identified and carried avrLm1-2-3-4-9-11-LepR1LepR2-S-AvrLm5-6-7, which is virulent on B. napus genotypes with different resistance genes (except $R \operatorname{lm} 7$ ). This isolate was considered the ideal candidate, not only for elucidating the role of Avr effectors in pathogenicity, but also with which to examine the role of $A v r-R$ interaction in the B. napus host defense. For breeding programs, we were expecting an ideal isolate that does not carry any of the identified Avr genes in L. maculans, and therefore, can be adopted for new resistance gene identification in Brassica genotypes. Brassica genotype showing incompatible reaction to this isolate should have a corresponding novel resistance $R$ gene. AvrLm4-7 gene has been cloned and determined by a single nucleotide mutation in coding region, where codon ${ }^{358}$ shifted from " $C$ " to " $G$ " conferring recognition to $R \operatorname{lm} 7$ and $R \operatorname{lm} 4 / 7$ in B. napus, respectively (Parlange et al., 2009). To knock out AvrLm7 gene for resistance screening in Brassica germplasm, we generated a deletion in AvrLm7 by employing CRISPR/Cas9. To validate this gene deletion, we analyzed the pathogenicity of both UMAvr7 (wild type) and the umavr7 (CRISPR mutant) on the corresponding B. napus cultivars 01-23-2-1 (with $R \operatorname{lm} 7$ ) and Westar (no R gene), along with sequencing. The main objective of this study is to obtain a suitable isolate (mutant) altering the pathogenicity on B. napus $R \operatorname{lm} 7$ genotype. We also evaluated an additional 123 B. napus genotypes to putatively reveal any unknown $R$ genes screened by this isolate.

\section{MATERIALS AND METHODS}

\section{Leptosphaeria maculans Isolate Preparation, DNA Extraction and PCR Assays}

A L. maculans isolate (DS103) (hereafter named UMAvr7) collected in a previous study, and carrying the AvrLm5-6-7 gene, was identified (Fernando et al., 2018). The isolate was grown on V8 agar juice medium amended with $0.0035 \%(w / v)$ streptomycin sulfate under continuous florescent light at room 
temperature for one week, before being subjected to a second round of single-pycnidiospore isolation to obtain a pure culture. The pycnidiospore and mycelia on the plate were flooded with $3 \mathrm{~mL}$ of sterile distilled water, collected and used for subsequent inoculum preparation and DNA extraction.

Genomic DNA was extracted from a mixture of pycnidiospore and mycelia suspensions of purified L. maculans isolates, using the modified CTAB method, as described by Liban et al. (2016). We used PCR to confirm the presence or absence of cloned avirulence genes including AvrLm1 (Gout et al., 2006), AvrLm2 (Ghanbarnia et al., 2015), AvrLm4-7 (Parlange et al., 2009), AvrLm5-9 (Van de Wouw et al., 2014; Ghanbarnia et al., 2018), AvrLm6 (Fudal et al., 2007), AvrLm11 (Balesdent et al., 2013), and AvrLm3 (Plissonneau et al., 2016). The primers used are listed in Supplementary Table $\$$.

\section{Phenotypic Characterization of the AvrLm4-7 Allele in the UMAvr7 L. maculans Isolate}

Avirulence gene profiles of $L$. maculans isolates were determined by inoculating the isolates onto a set of Brassica differentials harboring differently related resistance $(R)$ genes (Supplementary Table S2). Seeds of eleven Brassica differential genotypes were each sown into 96-cell flats filled with ProMix BX (Premier Tech, Rivière-du-Loup, Québec, Canada) and grown in a controlled growth chamber at $16 / 21^{\circ} \mathrm{C}$ (night/day) with a $16-\mathrm{h}$ daily photoperiod.

The concentration of harvested spores was adjusted to $2 \times 10^{7}$ spores $/ \mathrm{mL}$ for the cotyledon inoculation test. Cotyledons from each seedling (7 days old) were punctured on four lobes and inoculated with a droplet of $10 \mu \mathrm{L}$ inoculum, as previously described by Zhang et al. (2016). A minimum of six plants of each genotype were used for the inoculation test. Disease symptoms in infected cotyledons were evaluated at $14 \mathrm{dpi}$ (day post-inoculation) using a rating scale of $0-9$, based on lesion size, necrosis/chlorosis and the presence of pycnidia (Williams and Delwiche, 1979; Zhang et al., 2016).

\section{Generation of Mutant Isolate umavr7}

To generate the vector pKHT332, used to deliver the CRISPR/Cas9 system into L. maculans, a fragment containing Aspergillus nidulans tef 1 promoter, Cas 9 gene and Aspergillus nidulans tef1 terminator was amplified from a pFC334 plasmid (Nødvig et al., 2015) and then inserted into pKHT332 via InFusion cloning (Clontech, Takara Bio, United States) (Figure 1). The open reading frame of AvrLm4-7 was submitted to the CRISPR Optional Target Finder ${ }^{1}$ to search sgRNA sequences. We selected sgRNA showing at least five mismatched base pairs with other non-targeted sequences as the candidate sequence to BLAST against the L. maculans genome. We generated sgAvrLm4-7:pKHT332 by inserting the AvrLm47 sgRNA gene together with the gdpA promoter and trpC terminator into pKHT332 (restriction enzyme cutting by $\mathrm{PacI}$ ) by integrating two PCR fragments amplified from plasmid pFC334 via In-Fusion cloning (Takara Bio, Japan) (Figure 1), as described by Nødvig et al. (2015). PfuTurbo Cx Hotstart polymerase (Stratagen) was employed for amplification during

\footnotetext{
${ }^{1}$ http://tools.flycrispr.molbio.wisc.edu/targetFinder/
}

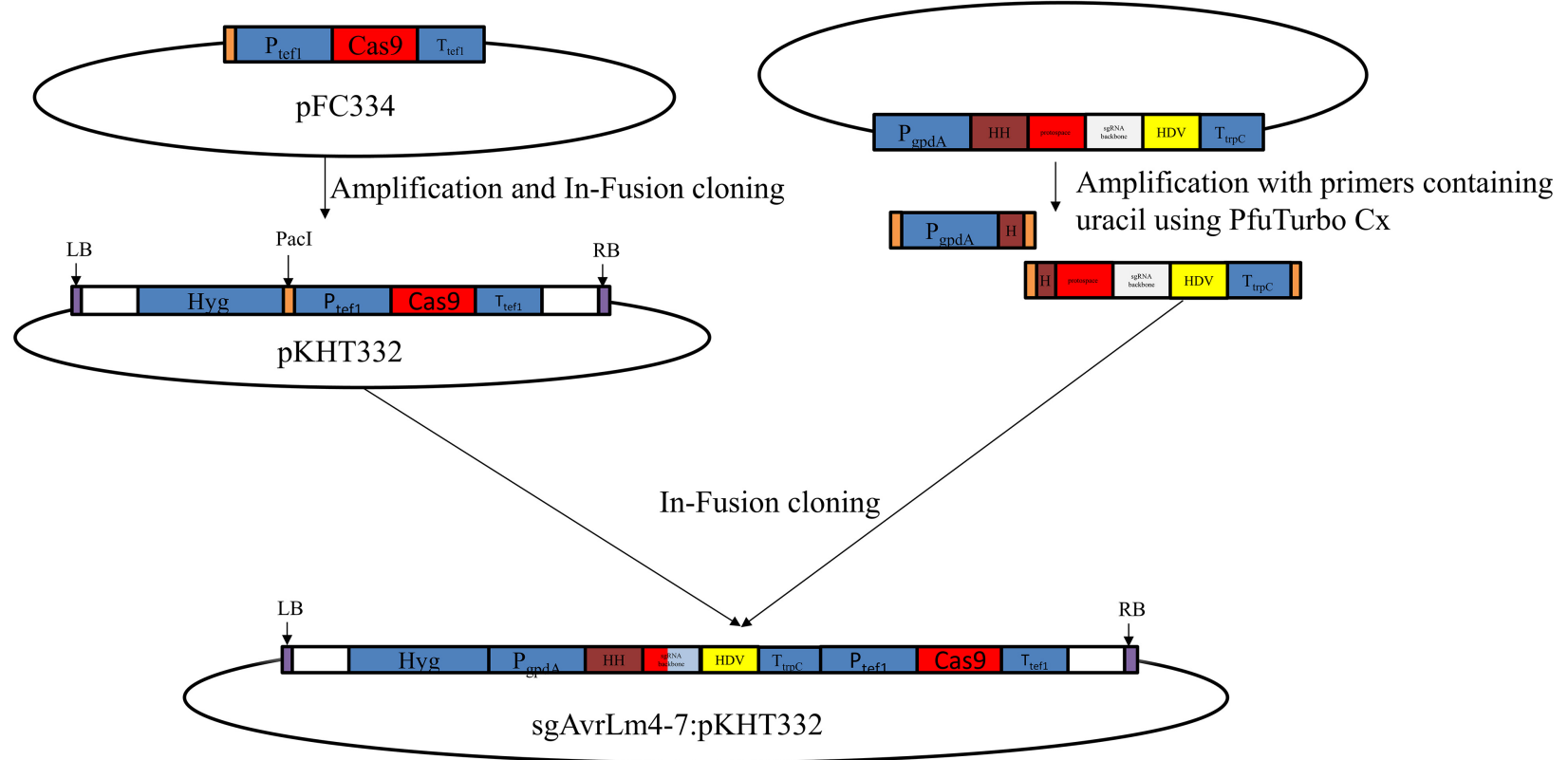

FIGURE 1 | Diagrammatic overview of the establishment of CRISPR/Cas9 mediate system in Leptosphaeria maculans AvrLm4-7 gene via in-fusion cloning strategy. Briefly, a fragment containing Aspergillus nidulans tef1 promoter, Cas9 gene, and Aspergillus nidulans tef1 terminator was amplified from a pFC334 plasmid and then inserted into pKHT332 via In-Fusion cloning. sgAvrLm4-7:pKHT332 by inserting the AvrLm4-7 sgRNA gene together with the gdpA promoter and trpC terminator into pKHT332 (restriction enzyme cutting by Pacl) by integrating two PCR fragments amplified from plasmid pFC334 via In-Fusion cloning. 
In-Fusion cloning. Sequences of primers used for the constructs are shown in Supplementary Table S1. Agrobacteriummediated transformation of UMAvr7 was conducted, as previously described by Gardiner and Howlett (2004). Briefly, the Agrobacterium with binary vector were cultured from a single colony and co-cultured with prepared L. maculans (DS103) spore solution filtered through sterile Miracloth and funnel. Then, the co-culture of Agrobacterium and L. maculans was poured to $25 \mathrm{~mL}$ IM plates (induction media) with AS (acetosyringone). After $48 \mathrm{~h}$ incubation at $22^{\circ} \mathrm{C}$ in the dark, the plates were overlaid with $25 \mathrm{~mL} 10 \% \mathrm{~V} 8$ medium with $100 \mu \mathrm{g} / \mathrm{mL}$ hygromycin. The plates were sealed and placed in a growth cabinet with lights at $22^{\circ} \mathrm{C}$ to enable $L$. mauclans mutant growth for 5-10 days. The generated mutants were grown on the V8 medium with hygromycin for positive colony selection. A single pycnidiospore was selected, cultured, and used for the following experiments.

\section{CRISPR/Cas9 Efficiency and Off-Targets Analysis}

A total of 100 candidate isolates were generated and selected for pathogenicity testing to validate the actual strains harboring a potential deletion in AvrLm7. The cotyledon inoculation method was performed as described above. The isolates were maintained on V8 juice, and pycnidiospores were collected for genomic DNA extraction. To confirm the sequence variation in targeted sgRNA regions, candidate isolates were amplified and sequenced with Seq4-7 F/R primers (Supplementary Table S1). Sequence alignment was conducted by SeqMan in DNAstar ${ }^{2}$.

To analyze the potential off-targets of sgRNAAvrLm4-7-Cas9, the sgRNA sequence was employed to search the L. maculans genome database ${ }^{3}$. Sequence identity of at least $10 \mathrm{bp}$ to sgRNA was selected to identify potential off-targets. Primers were designed for amplifying and sequencing the off-target regions in both wild type (UMAvr7) and mutant (umavr7) isolates (Supplementary Table S1).

\section{Reverse Transcription PCR for Expression Analysis of AvrLm7}

The wild-type (UMAvr7) and mutant (umavr7) isolates were inoculated on cotyledons of 7-day-old seedlings of $B$. napus genotypes Westar (No $R$ gene) and 01-23-2-1 ( $R \operatorname{lm} 7$ gene). RNA was extracted from $100 \mathrm{mg}$ of cotyledons sampled from 1 day and 11 days post inoculation using Plant RNA Reagent (Invitrogen, Carlsbad, CA, United States). The first strand cDNA was synthesized from one microgram of total RNA from each sample using RevertAid First Strand cDNA Synthesis Kit according to the manufacturer's instructions (Thermo Scientific, Waltham, MA, United States). Reverse transcription PCR (RTPCR) was performed with the primers designed from coding region of $A v r L m 7$ gene by 30 cycles of $94^{\circ} \mathrm{C}$ for $30 \mathrm{~s}, 58^{\circ} \mathrm{C}$ for $30 \mathrm{~s}$, and $72^{\circ} \mathrm{C}$ for $30 \mathrm{~s}$. Three biological replicates were sampled for RT-PCR analysis. B. napus actin gene was used as a control.

${ }^{2}$ https://www.dnastar.com/software/molecular-biology/

${ }^{3}$ https://genome.jgi.doe.gov/pages/blast-query.jsf?db=Lepmu1

\section{Pathogenicity Evaluation of L. maculans Isolates UMAvr7 and umavr7}

To compare the disease severity of the wild-type isolate UMAvr7 and its mutant (umavr7) generated from the CRISPR/Cas9 system, both isolates were inoculated onto punctured cotyledons of B. napus genotypes Westar and 01-23-2-1 seedlings 7 days after seeding, as described above. Lesion sizes were quantified at $14 \mathrm{dpi}$ in Assess 2.0 (American Phytopathological Society, St. Paul, MN, United States). A total of six plants with 24 lesions were quantified and the experiments were repeated once. Analysis of variance (ANOVA) were performed using SAS Version 9.4 (SAS Institute, Inc., Cary, NC, United States).

\section{In situ Histochemical Detection of Hydrogen Peroxide}

Hydrogen peroxide levels in the seedling cotyledons of B. napus cultivars Westar and 01-23-2-1 inoculated with wild-type and mutant isolates were detected via 3,3-diaminobenzidine (DAB) staining. The staining solution was prepared by dissolving $50 \mathrm{mg}$ DAB in $50 \mathrm{~mL}$ sterile $\mathrm{H}_{2} \mathrm{O}(1 \mathrm{mg} \mathrm{mL}-1$ DAB solution), with the $\mathrm{pH}$ adjusted to 3.0 with $0.2 \mathrm{M} \mathrm{HCl}$. After immersion in the DAB staining solution in the dark for 6 hours at room temperature, the stained cotyledons were then cleared using bleaching solution (ethanol: acetic acid: glycerol $=3: 1: 1$ ). Hydrogen peroxide $\left(\mathrm{H}_{2} \mathrm{O}_{2}\right)$ oxidizes $D A B$ in the presence of peroxidases and produces a reddish brown precipitate (Daudi and O'Brien, 2012).

\section{Broadening the Genetic Resources of L. maculans Resistance in Brassica napus by Inoculation With Mutant Isolate umavr7}

A collection of 123 B. napus genotypes, consisting of varieties and advanced breeding lines, were selected for the pathogenicity test using the umavr7 isolate under greenhouse conditions (Fernando et al., 2018). These B. napus genotypes included winter types and semi-winter types and were derived from the major canola growing areas of China. All B. napus genotypes were subjected to cotyledon inoculation tests with at least six plants of each genotype. The inoculum preparation, inoculation methods, rating, and resistance analysis used were described as above.

\section{RESULTS}

\section{Genotype Confirmation of the}

\section{L. maculans UMAvr7 Isolate}

A L. maculans isolate (DS103), termed UMAvr7, was originally identified in a previous study during a canola disease survey in Manitoba, Canada (Fernando et al., 2018). PCR analysis of this isolate, applied to determine the Avr gene profile, indicated that only the AvrLm5, AvrLm6, and AvrLm4-7 genes could be positively amplified. Transcriptome analysis of inoculating this isolate on susceptible and resistant $B$. napus genotypes by dualRNA sequencing also indicated that none of the AvrLm1, 2, 3, and 
11 genes were expressed, except AvrLm5, AvrLm6, and AvrLm47 genes (unpublished transcriptome data). Taken together, UMAvr7 is lacking most known avirulence genes that interact with the resistance genes from $B$. napus and was considered an ideal candidate for creating a more virulent $L$. maculans strain.

To confirm the PCR genotyping, UMAvr7 was inoculated on a set of Brassica differentials carrying the resistance genes $R \operatorname{lm} 1$, 2, 3, 4, 6, 7, 9, and LepR1, R2, R3 to profile the avirulence genes. The results revealed that UMAvr7 caused disease symptoms on all tested Brassica differentials with the exception of the B. napus genotype 01-23-2-1 $(R \operatorname{lm} 7)$ and $B$. juncea genotype Forge (Rlm6) (Supplementary Figure S1). The disease rating scores ranged between 7 and 9 for the cotyledons of the differentials that displayed a susceptible reaction. As expected, B. napus genotype 01-23-2-1 $(R \operatorname{lm} 7)$ and B. juncea genotype Forge $(R \operatorname{lm} 6)$ displayed high levels of resistance (disease rating score of 1.23 and 2.67) to UMAvr7 (Supplementary Figure S1). Currently, we do not have a proper $B$. juncea genotype carrying $R \operatorname{lm} 5$ to characterize the phenotype consistent with the AvrLm5 genotype in the isolate. However, the main objective of this study was to mutate the AvrLm7 gene in the isolate that interacts with the $R \operatorname{lm} 7$ gene from $B$. napus. Therefore, this isolate can be adopted and used to broaden genetic resistance resources from $B$. napus genotypes.

\section{Production of a Mutant Isolate Using the CRISPR/Cas9 System}

A total of 100 mutant isolates were picked from the growth medium after Agrobacterium transformation. Through PCR assays, no amplification of AvrLm1, 2, 3, 11 was observed in the collected mutant. As expected, positive PCR products could be obtained from the genomic DNA of all the mutants, with primers specific to AvrLm4-7.

For the 100 selected isolates, the nucleotides were edited near the genomic region of the guide sgRNA targeting Cas9 in 32 mutant isolates (Supplementary Figure S2). Only six mutants featured $2-8$ bp deletions and the remaining mutant isolates were found to have single nucleotide insertion/deletions or substitutions (Supplementary Figure S2). The AvrLm4-7 gene had two exons in L. maculans isolates (Parlange et al., 2009); the edited regions in the first exon produced significant amino acids variations in coding regions including deletions (Supplementary Figure S3). To screen the mutants, all the transformed isolates were inoculated onto the set of Brassica differentials to phenotype their pathogenicity. The disease rating scored from 1 to 3 in B. napus $R \operatorname{lm} 7$ genotype caused by other 31 mutants, indicating that there was no shift from avirulence to virulence on the genotype 01-23-2-1, which carries the $R \operatorname{lm} 7$ gene that interacts with AvrLm7 (Supplementary Table S3).

Among these mutants, one isolate (Mu3), hereafter termed as umavr7, when inoculated onto B. napus genotype 01-232-1 $(R \operatorname{lm} 7)$, generated large disease lesions similar to those observed in the susceptible Westar genotype (Figure 2A), indicating a shift from avirulence to virulence. The mutant isolate, umavr7, produced a significantly large lesion on 01-23-21 , which was similar in size to lesions observed after inoculation on Westar (Figure 2B). An 8 bp deletion (TCAAGGCA) in umavr7, overlapping the sgRNA region, was confirmed by DNA sequencing. This deletion caused the amino acid change in the coding region of the AvrLm4-7 gene in L. maculans UMAvr7 (Figure 3). Transcriptomes analysis in B. napusL. maculans pathosystem of compatible and incompatible interaction on $R \operatorname{lm} 7$ genotype using wild type (UMAvr7) and the mutant isolate (umavr7), respectively, indicated that there is no AvrLm7 expression in transcription data generated from umavr7 inoculation (unpublished transcriptome data). Reverse transcription (RT)-PCR assays also indicated that there is no AvrLm7 expression in mutated isolate, and in Westar/01-23-2-1 $(R \operatorname{lm} 7)$ genotypes inoculated with mutant isolate at 1 and $11 \mathrm{dpi}$, respectively (Figure 4).

To eliminate the possibility of off-target editing by CRISPR/Cas9 system, we searched six potential off-target genomic regions from the determined genome sequence of L. maculans. After PCR amplification and sequencing of both wild type (UMAvr7) and mutant isolate (umavr7), there was no nucleotide polymorphism in all six potential off-target regions (Supplementary Table S4 and Supplementary Figure S4), indicating that the CRISPR/Cas9 system developed in this study specifically edited the genomic region of AvrLm4-7 gene. Taken together, the pathogenicity of the mutant isolate umavr7 shifted from avirulent to virulent on the $B$. napus genotype harboring the $R \operatorname{lm} 7$ gene, based on the cotyledon inoculation test and PCR genotyping validation. Therefore, the mutant isolate that caused significant disease symptoms on $B$. napus genotypes with known $R$ genes can be considered a virulent isolate for $B$. napus genotypes resistance screening.

\section{$\mathrm{H}_{2} \mathrm{O}_{2}$ Accumulation of Cotyledons in Response to Mutant Isolate}

In situ detection of $\mathrm{H}_{2} \mathrm{O}_{2}$ was detected in Westar and 01-23-2-1 at $1,3,7$, and 11 dpi. Upon DAB staining, the $\mathrm{H}_{2} \mathrm{O}_{2}$ accumulation was not shown to be obviously different between cotyledons infected with UMAvr7 and umavr7 at an earlier stage (1-7 dpi) in both genotypes, i.e., from $1 \mathrm{dpi}$ to $7 \mathrm{dpi}$. At $11 \mathrm{dpi}$, the cotyledons inoculated with UMAvr7 and umavr7 displayed a stronger reddish coloration around decayed tissue on Westar. However, in cotyledons of resistant genotype 01-23-2-1, UMAvr7 just caused limited accumulation of hydrogen peroxide around the wound site compared to mutant isolate umavr7 (Figure 5). These results demonstrated that hydrogen peroxide in cotyledons accumulate differently in response to infection with UMAvr7 and umavr7. For example, in resistant genotype 01-23-2-1, the hydrogen peroxide accumulation increased when infected with the mutant isolate at a late stage of infection.

\section{B. napus Germplasms Resistance Screening}

Based on disease rating scores averaged from at least six plants with 24 wounded lobes, 115 B. napus genotypes displayed susceptible disease symptoms (6.1-9.0), meaning the mutant isolate was virulent on most of the genotypes carrying $R \operatorname{lm} 1$, $R \operatorname{lm} 2, R \operatorname{lm} 3$, and $R \operatorname{lm} 4$, or those without any of the known $R$ genes (Supplementary Table S5). Two B. napus genotypes, 


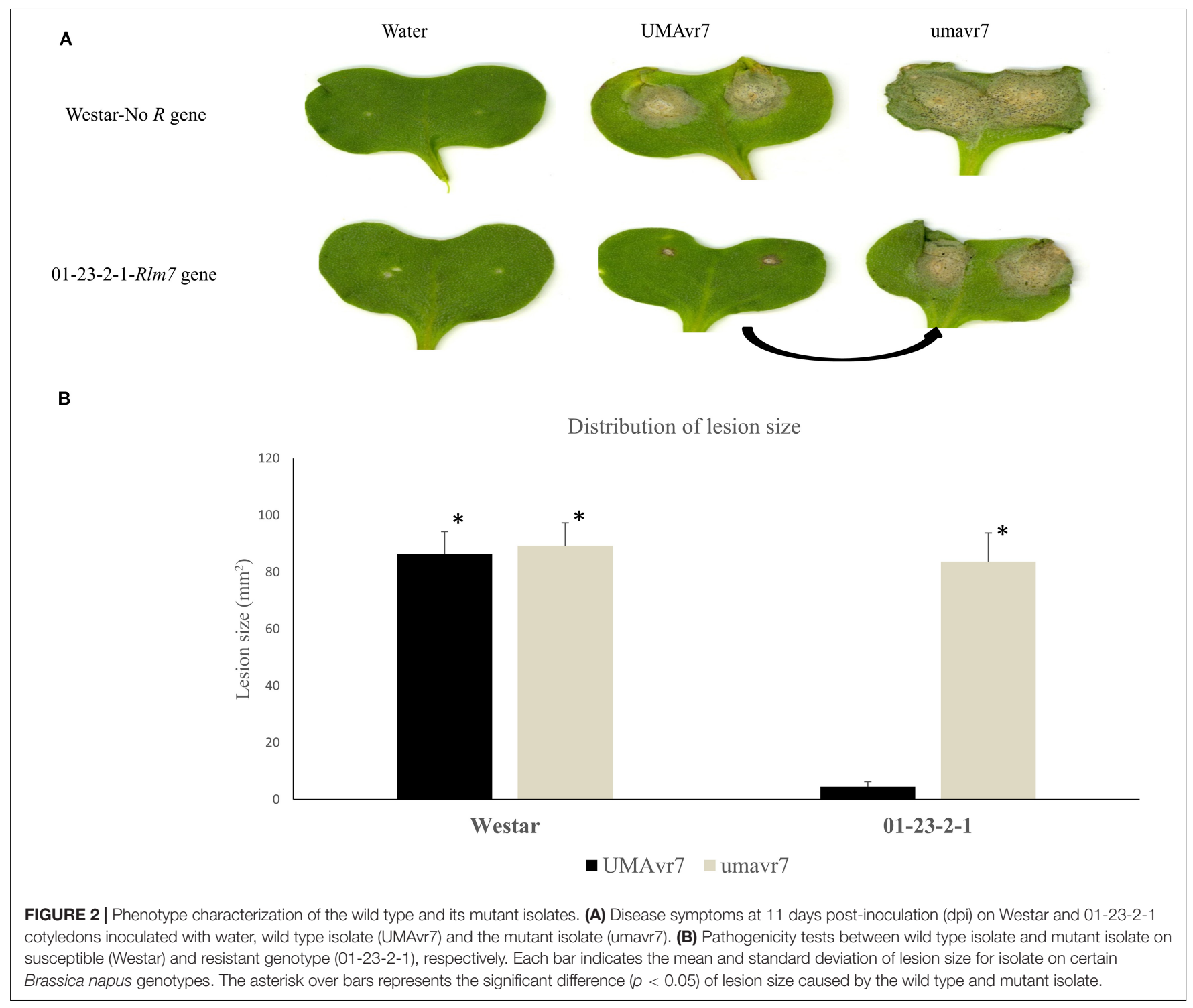

1055 and 8013, carrying $R \operatorname{lm} 2 /$ unknown and $R \operatorname{lm} 3$, respectively, showed intermediate resistance to the mutant isolate (umavr7). Interestingly, six genotypes, including 1056, 8011, 8037, HP49, CC01, and HP15, showed clear resistance to the mutant isolate (umavr7). Among the six genotypes, 8011, HP49 and HP15 were found to carry an unknown resistant gene, when characterized in a previous study (Zhang et al., 2017). The other three germplasm, 1056, 8037, and CC01, were found to have Rlm2/unknown, $R l m 2 / R l m 3 /$ unknown, and $R l m 3 /$ unknown genes, respectively (Zhang et al., 2017). Theoretically, any B. napus genotype showing resistance to the mutant isolate has a high possibility of harboring a novel resistant gene, as the isolate lacks all of the identified avirulence genes that interact with resistance genes from $B$. napus. Our results indicate that the mutant umavr7 isolate, which has avirulence gene (AvrLm7) knocked out, can be used to rapidly screen the unknown resistance gene in B. napus instead of using a large number of differential L. maculans isolates.

\section{DISCUSSION}

This paper reports the generation of an avirulence gene (AvrLm7) mutation in a $L$. maculans strain, using the gene-editing tool CRISPR/Cas9. The mutant isolate caused significant disease symptoms in all the $B$. napus genotypes carrying known $R$ genes, especially in 01-23-2-1, in which disease pathogenicity changed from avirulent in the wild-type isolate to virulent in the mutant. We applied the mutant umavr7 isolate to screening novel $R$ genes in $B$. napus genotypes.

Currently, breeding programs aimed at developing resistance to blackleg rely on the gene-for-gene interaction model between Brassica species and L. maculans, with the latter carrying the avirulence gene that selects the corresponding $R$ gene in the former. To date, a total of 19 major resistance genes have been identified and reported in several Brassica species, providing information on race-specificity of $L$. maculans resistance. In these studies, L. maculans isolates carrying different Avr genes were 


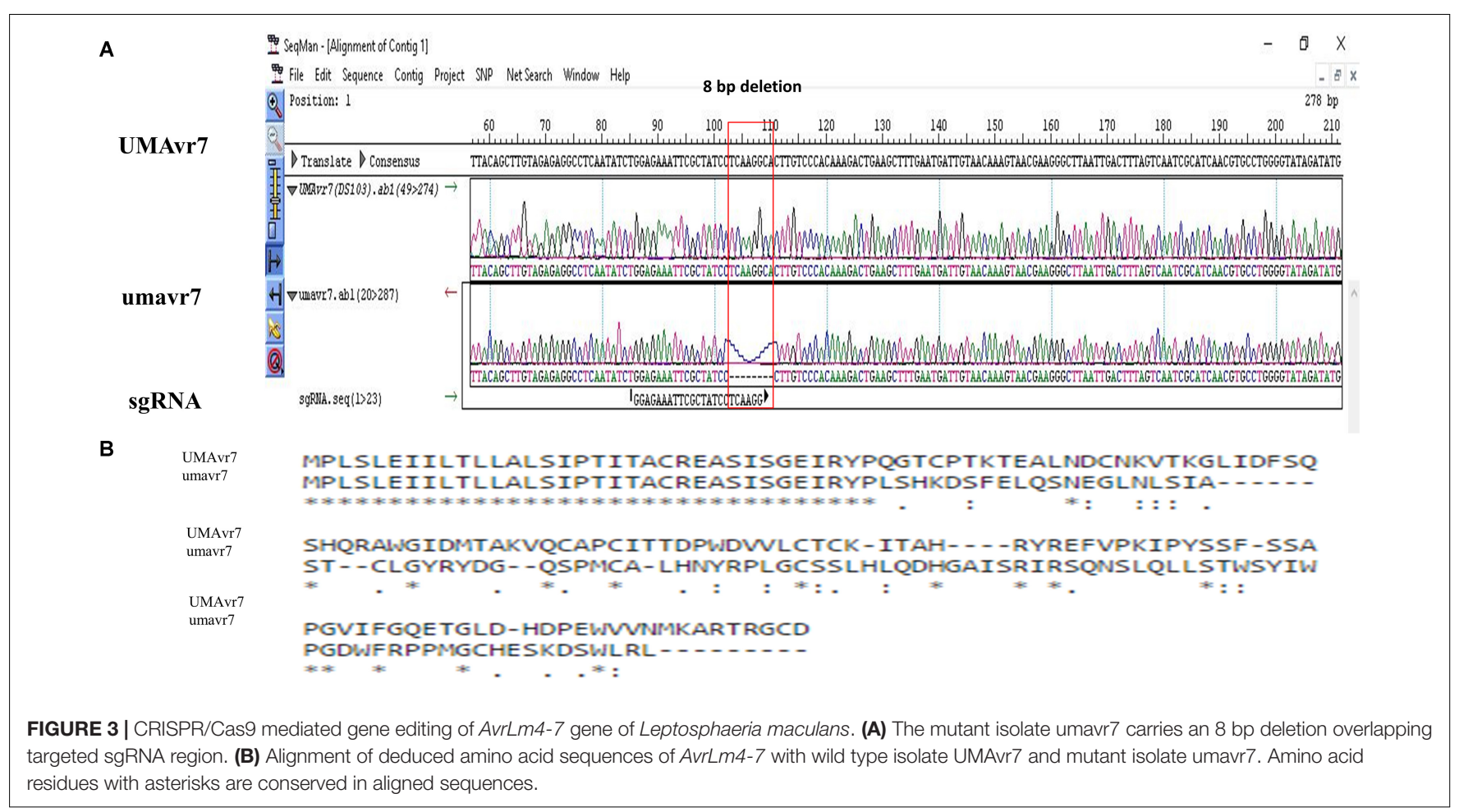

inoculated onto plants to phenotype disease severity, including "P042" (AvrLm1, AvrLmS) (Larkan et al., 2013) and "165" (avrLm1, AvrLm2, avrLm3) (Larkan et al., 2015), which were adopted for $R \operatorname{lm} 1$ and $R \operatorname{lm} 2$ gene identification. Two isolates harboring different $A v r$ gene race structures were inoculated on Brassica crops, to evaluate blackleg disease resistance and identify corresponding resistance genes. Because the isolates used carried a different number of $A v r$ genes, their pathogenicity on different canola genotypes varied from virulence to avirulence. Therefore, broadening the genetic bases of resistance through interspecific or intergeneric hybridization to improve resilience against blackleg disease is an important strategy in canola plant breeding. To address this issue, generating a virulent strain of L. maculans, which can cause blackleg disease in B. napus (including those harboring a specific $R$ gene), is essential for screening resistant phenotypes in a breeding program. In a disease survey conducted in Western Canada, 180 Avr gene races were identified, with several major races, including AvrLm24-5-6-7-11, AvrLm2-4-5-6-7-11-S, and AvrLm1-4-5-6-7-11-(S), accounting for approximately $25 \%$ of the population (Fernando et al., 2018). However, these isolates can only be used to screen the comparatively lower level resistant Brassica genotypes that show specific resistance to the designated L. maculans isolates with corresponding Avr genes. In contrast, the mutant isolate umavr7, generated via a genome editing tool, theoretically carries none of the known Avr genes and can cause all B. napus genotypes (with $R$ gene resistance) to exhibit disease symptoms. Therefore, this mutant isolate has the potential for a wide range of uses in the selection of $B$. napus genotype with strong resistance to blackleg disease.
B. napus (AACC, $2 n=38$ ) is a relatively young species derived from spontaneous interspecific hybridization between Brassica rapa (AA, $2 n=20$ ) and Brassica oleracea (CC, $2 n=18$ ). In addition, embryo rescue has been applied successfully to overcome the barrier of interspecies self-incompatibility and to increase the rate of crossing (Tonguc and Griffiths, 2004), by manipulation of the extremely high level of resistance exhibited by $B$. napus and resynthesized lines to mutant strains of L. maculans through breeding. In our investigation, the mutant isolate umavr7 was further evaluated on 123 B. napus genotypes, only six of which showed blackleg disease resistance. As is our understanding, these six B. napus genotypes should harbor novel $R$ gene(s). This result is consistent with that previously reported by Zhang et al. (2017), who found 12 accessions exhibiting unknown $R$ genes in greenhouse and field trials. However, in comparison to the identification of $B$. napus seedling or adult plant germplasm resistance to blackleg disease, using 30 different L. maculans isolates, carried out by Zhang et al. (2017), the application of the mutated isolate umavr7 enables an easier and more rapid route to identify resistant genotypes, as well as the identification of genotypes carrying novel/unknown $R$ gene(s). These resistant genotypes can then be used for blackleg disease breeding and novel $R$ gene identification. In the case of the 19 identified $R$ genes, most have been mapped to the " $A$ " genome of B. napus or B. rapa (Rlm1-4, Rlm7 and LepR1-4) via linkage mapping. Introducing the genes conferring resistance to L. maculans into B. rapa (A genome), and B. oleracea (C genome) is another option for enlarging the gene pool for canola disease breeding via interspecific crossing. Individuals of B. rapa or $B$. oleracea accessions, screened by mutant isolates, can then be 


\section{1-23-2-1 and Pathogen dual RNA-seq Westar and Pathogen dual RNA-seq}
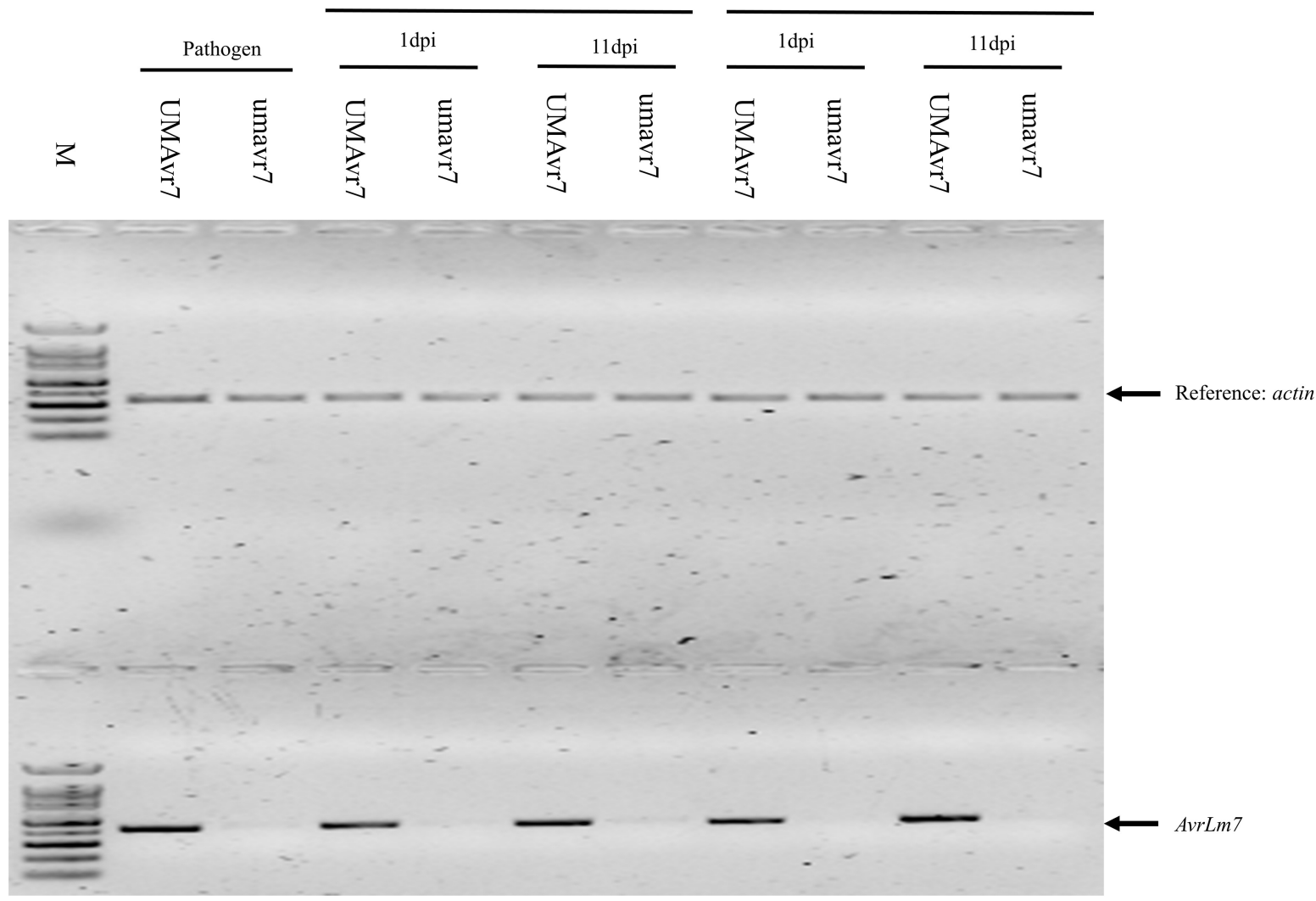

FIGURE 4 | Reverse transcription (RT)-PCR analysis of AvrLm7 gene in wild-type and mutant isolates only, and in Westar/01-23-2-1 genotypes inoculated with UMAvr7 and umavr7, respectively, at $1 \mathrm{dpi}$ and $11 \mathrm{dpi}$. Leptosphaeria maculans actin was used as a control to quantify equal RNA using.

crossed and resynthesized into amphidiploid B. napus plants with extremely high resistance to blackleg disease. The main objective of this study was to obtain a virulent isolate that can cause disease on the $B$. napus germplasm carrying resistance genes. Then, the isolate can be adopted for breeding resistance screening.

Previously, $\mathrm{H}_{2} \mathrm{O}_{2}$ accumulation during L. maculans infection has been reported by Nováková et al. (2016). They found that the isolate having AvrLm4-7 in the interaction led to less accumulation of hydrogen peroxide at later stages [ 8 and 10 days after inoculation (dai)]. We found similar results in that the wild type isolate UMAvr7 carrying AvrLm7 caused a decreased accumulation of $\mathrm{H}_{2} \mathrm{O}_{2}$ in incompatible interaction of cotyledons in $R \operatorname{lm} 7$ genotype, while the mutant isolate (avrLm7) with knocked out AvrLm 7 gene showed increased accumulation at 7 and $11 \mathrm{dpi}$. This increased accumulation of $\mathrm{H}_{2} \mathrm{O}_{2}$ was similar to the compatible reactions on the susceptible genotype "Westar" caused by both wild type and mutant isolates. This demonstrates that $\mathrm{H}_{2} \mathrm{O}_{2}$ plays important roles in the defense response to L. maculans infection. Nováková et al. (2016) also indicated that rbohF encoding one of the NADPH oxidases for ROS (reaction oxygen species) production in the apoplast showed decreased transcription in cotyledons infected with the isolate carrying with AvrLm4-7. One effector gene pep1 from the biotrophic pathogen Ustilago maydis has been reported to inhibit the maize peroxidase
POX12 in the apoplast (Hemetsberger et al., 2012). However, we cannot prove that the AvrLm7 will affect the accumulation of $\mathrm{H}_{2} \mathrm{O}_{2}$ similar to pep1-regulation manner without investigation of purified AvrLm7 protein effects on the ROS production; or the SA (salicylic acid) and ET (ethylene) signaling affected by ROS production or $\mathrm{H}_{2} \mathrm{O}_{2}$ accumulation will enhance the hypersensitive reaction (HR) to establish the defense when the plant infected with isolates having effector genes (Draper, 1997; de Jong et al., 2002). Therefore, the hypothesis of that the presence of AvrLm7/AvrLm4-7 will reduce the accumulation of ROS and drive the HR in the B. napus-L. maculans pathosystem through affecting the SA and ET signaling pathways needs to be further studied. However, our observation in this study provides insights in that the isolates with the same genetic background will cause different accumulation levels of $\mathrm{H}_{2} \mathrm{O}_{2}$ by knocking out the effector gene AvrLm7 and therefore, show avirulence and virulence on the B. napus genotype with corresponding resistance gene $(R \operatorname{lm} 7)$. Traditional approaches used to determine the functions of uncharacterized or characterized fungal genes are typically based on the identification of phenotypic expression, or the homologous recombination-mediated switching on or off of a particular gene. However, the latter method of gene targeting is not particularly efficient in fungi and is limited by incompatible mutagenesis screening on a genome-wide scale. More recently, 


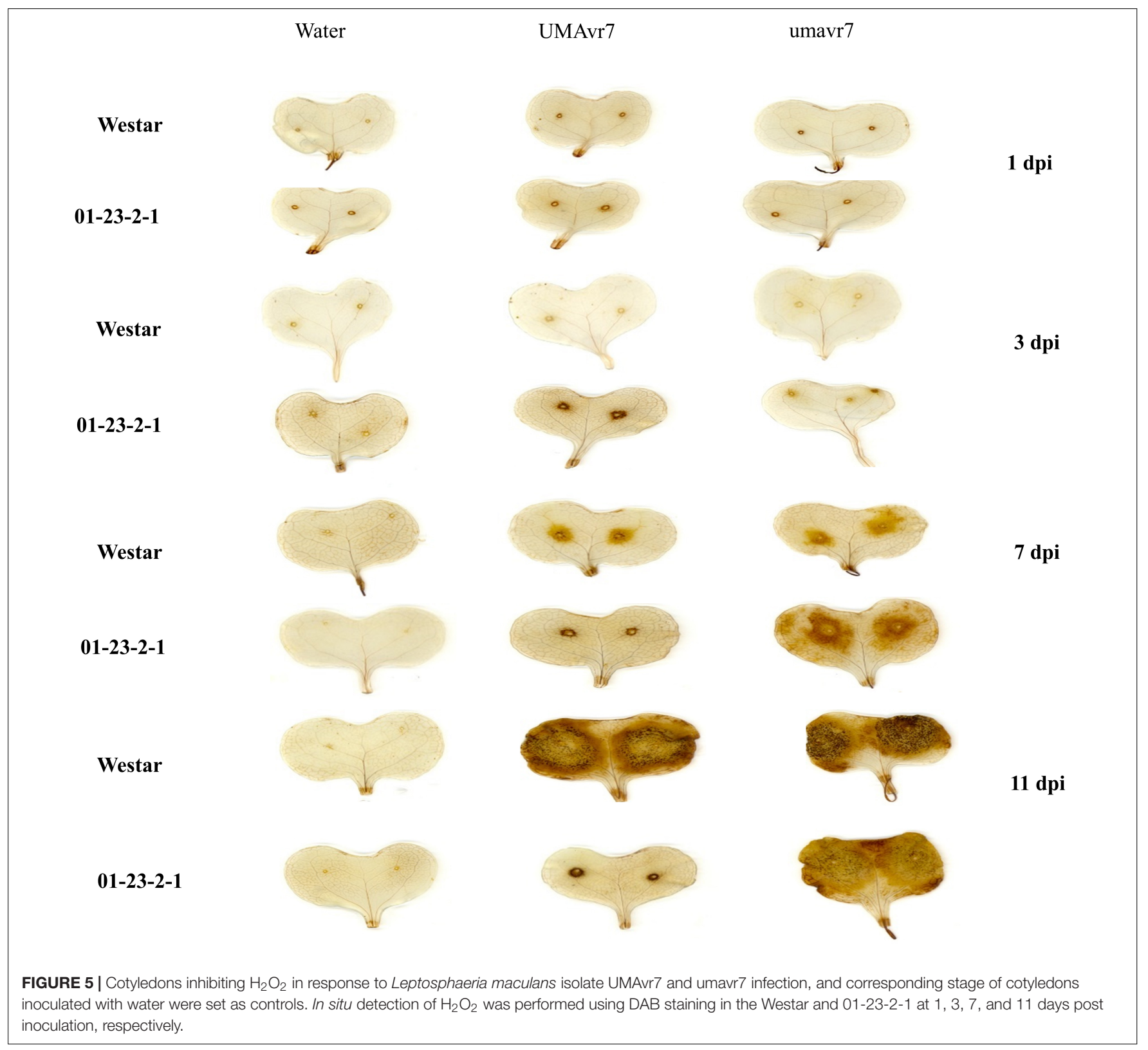

the CRISPR/Cas9 system has been widely adopted to disrupt target genes in fungi and bacteria (Takayuki et al., 2015; Deng et al., 2017; Idnurm et al., 2017; Schuster et al., 2018).

This method represents a very important technological advance in the simultaneous disruption of functional genes/gene families to investigate their specific contribution to pathogen virulence. The L. maculans isolate UMAvr7, used in the present study, carried the AvrLm7 gene, which confers a high level of virulence to canola genotypes, with the exception of those genotypes carrying the $R \operatorname{lm} 7$ gene. To obtain a mutant isolate that can be used in material screening and breeding, the AvrLm7 gene must be mutated and its pathogenicity altered. Here we adapted CRISPR/Cas9 to disrupt a specific gene (AvrLm7) in the UMAvr7 isolate without the need for resistance markers.
The efficiency of this CRISPR/Cas9-mediated AvrLm7 gene editing was quite high in progeny derived from a single transformant, as demonstrated by sequencing and phenotyping, with $32 \%$ mutant progeny produced from transformation of a single isolate and with different sequence variations in the selected candidate isolates. For the editing of specific genes, CRISPR/Cas9 works easily and quickly to target the gene used to produce mutants for subsequent function analysis. Another advantage of the CRISPR/Cas9 technique is that it offers the possibility of disrupting several Avr genes simultaneously, by using multiple sgRNAs or a single sgRNA molecule targeting a region that is highly conserved across members of a gene family. A future interest for our research group is to use the CRISPR/Cas9 molecular tool to investigate the difference 
in virulence provided by a single $A v r$ gene mutation and by multiple $A v r$ gene mutations. In addition, the genomic and transcriptional profiling of wild-type and mutant isolates, as well as plant defense responses to infection, should provide a deeper understanding of the gene interactions between canola and the L. maculans pathogen.

\section{DATA AVAILABILITY STATEMENT}

All datasets presented in this study are included in the article/Supplementary Material.

\section{AUTHOR CONTRIBUTIONS}

$\mathrm{ZZ}$ and WF designed the experiments and wrote and revised the manuscript. ZZ, FL, and CS performed the experiments. $\mathrm{ZZ}, \mathrm{FL}$, and WF analyzed and interpreted the data. All authors contributed to the article and approved the submitted version.

\section{REFERENCES}

Ansan-Melayah, D., Balesdent, M. H., Delourme, R., Pilet, M. L., Tanguy, X., Renard, M., et al. (1998). Genes for race-specific resistance against blackleg disease in Brassica napus L. Plant Breed. 117, 373-378. doi: 10.1111/j.1439-0523. 1998.tb01956.x

Balesdent, M. H., Attard, A., Kuhn, M. L., and Rouxel, T. (2002). New avirulence genes in the phytopathogenic fungus Leptosphaeria maculans. Phytopathology 92, 1122-1133. doi: 10.1094/PHYTO.2002.92.10. 1122

Balesdent, M. H., Fudal, I., Ollivier, B., Bally, P., Grandaubert, J., Eber, F., et al. (2013). The dispensable chromosome of Leptosphaeria maculans shelters an effector gene conferring avirulence towards Brassica rapa. New Phytol. 198, 887-898. doi: 10.1111/nph.12178

Brooks, C., Nekrasov, V., Lippman, Z. B., and Eck, J. V. (2014). Efficient gene editing in tomato in the first generation using the clustered regularly interspaced short palindromic repeats/CRISPR-associated 9 system. Plant Physiol. 166, 1292-1297. doi: 10.1104/pp.114.247577

Cai, Y., Chen, L., Liu, X., Sun, S., Wu, C., Jiang, B., et al. (2015). CRISPR/Cas9mediated genome editing in soybean hairy roots. PLoS One 10:e136064. doi: 10.1371/journal.pone.0136064

Chèvre, A. M., Eber, F., This, P., Barret, P., Tanguy, X., Brun, H., et al. (1997). Selection of stable Brassica napus-B. juncea recombinant lines resistant to blackleg (Leptosphaeria maculans). Theor. Appl. Genet. 95, 1104-1111. doi: $10.1007 / \mathrm{s} 001220050669$

Daudi, A., and O'Brien, J. A. (2012). Detection of hydrogen peroxide by DAB staining in Arabidopsis leaves. Bio Protocol 2:e263.

Daverdin, G., Rouxel, T., Gout, L., Aubertot, J. N., Fudal, I., Meyer, M., et al. (2012). Genome structure and reproductive behaviour influence the evolutionary potential of a fungal phytopathogen. PLoS Pathogens 8:e1003020. doi: 10.1371/ journal.ppat.1003020

de Jong, A. J., Yakimova, E. T., Kapchina, V. M., and Woltering, E. J. (2002). A critical role for ethylene in hydrogen peroxide release during programmed cell death in tomato suspension cells. Planta 214, 537-545. doi: 10.1007/ s004250100654

Deng, H., Gao, R., Liao, X., and Cai, Y. (2017). CRISPR system in filamentous fungi: current achievements and future directions. Gene 627, 212-221. doi: 10.1016/j.gene.2017.06.019

Draper, J. (1997). Salicylate, superoxide synthesis and cell suicide in plant defence. Trends Plant Sci. 2, 162-165. doi: 10.1016/S1360-1385(97)01030-3

\section{FUNDING}

This work was funded by the Natural Sciences and Engineering Researching Council of Canada (NSERC) Discovery Program, The NSERC-CRD and the Growing Forward 2 canola cluster funds awarded to WF.

\section{ACKNOWLEDGMENTS}

We would like to thank Sakaria Liban in the collection and characterization of the blackleg isolate from the canola disease survey project. We thank Rasanie Padmathileke from our lab for allowing us to reference some of her transcriptome data to substantiate our work presented in this manuscript.

\section{SUPPLEMENTARY MATERIAL}

The Supplementary Material for this article can be found online at: https://www.frontiersin.org/articles/10.3389/fmicb. 2020.01969/full\#supplementary-material

Eber, F., Lourgant, K., Brun, H., Lode, M., Huteau, V., Coriton, O., et al. (2011). "Analysis of Brassica nigra chromosomes allows identification of a new effective Leptosphaeria maculans resistance gene introgressed in Brassica napus," in Proceeding of the 13th International Rapeseed Congress, Prague, 5-9.

Fernando, W. G. D., Zhang, X., and Amarasinghe, C. C. (2016). Detection of Leptosphaeria maculans and Leptosphaeria biglobosa causing blackleg disease in canola from Canadian canola seed lots and dockage. Plants 5:12. doi: 10.3390/ plants5010012

Fernando, W. G. D., Zhang, X., Selin, C., Zou, Z., Liban, S. H., McLaren, D. L., et al. (2018). A six-year investigation of the dynamics of Avirulence allele profiles, blackleg incidence, and mating type alleles of Leptosphaeria maculans population associated with canola crops in Manitoba. Canada. Plant Dis. 12, 790-798. doi: 10.1094/PDIS-05-17-0630-RE

Fitt, B. D. L., Brun, H., Barbetti, M. J., and Rimmer, S. R. (2006). World-wide importance of Phoma stem canker (Leptosphaeria maculans and L. biglobosa) on oilseed rape (Brassica napus). Eur. J. Plant Pathol. 114, 3-15. doi: 10.1007/14020-4525-5_1

Fudal, I., Ross, S., Gout, L., Blaise, F., Kuhn, M. L., Eckert, M. R., et al. (2007). Heterochromatin-like regions as ecological niches for avirulence genes in the Leptosphaeria maculans genome: map-based cloning of AvrLm6. Mol. Plant Microbe Interact. 20, 459-470. doi: 10.1094/MPMI-20-4-0459

Gardiner, D. M., and Howlett, B. J. (2004). Negative selection using thymidine kinase increases the efficiency of recovery of transformants with targeted genes in the filamentous fungus Leptosphaeria maculans. Curr. Genet. 45, 249-255. doi: 10.1007/s00294-004-0488-6

Ghanbarnia, K., Fudal, I., Larkan, N. J., Links, M. G., Balesdent, M. H., Profotova, B., et al. (2015). Rapid identification of the Leptosphaeria maculans avirulence gene AvrLm2 using an intraspecific comparative genomics approach. Mol. Plant Pathol. 16, 699-709. doi: 10.1111/mpp.12228

Ghanbarnia, K., Ma, L., Larkan, N. J., Haddadi, P., Fernando, W. G. D., and Borhan, M. H. (2018). Leptosphaeria maculans AvrLm9: a new player in the game of hide and seek with AvrLm4-7. Mol. Plant Pathol. 19, 1754-1764. doi: 10.1111/mpp.12658

Gout, L., Fudal, I., Kuhn, M. L., Blaise, F., Eckert, M., Cattolico, L., et al. (2006). Lost in the middle of nowhere: the AvrLm1 avirulence gene of the Dothideomycete Leptosphaeria maculans. Mol. Microbiol. 60, 67-80. doi: 10.1111/j.1365-2958. 2006.05076.x

Gugel, R. K., and Petrie, G. A. (1992). History, occurrence, impact and control of blackleg of rapeseed. Can. J. Plant Pathol. 14, 36-45. doi: 10.1080/ 07060669209500904 
Hayward, A., McLanders, J., Campbell, E., Edwards, D., and Barley, J. (2012). Genomic advances will herald new insights into the Brassica: Leptosphaeria maculans pathosystem. Plant Biol. 14, S1-S10. doi: 10.1111/j.1438-8677.2011. 00481.x

Hemetsberger, C., Herrberger, C., Zechmann, B., Hillmer, M., and Doehlemann, G. (2012). The Ustilago maydis effector Pep1 suppresses plant immunity by inhibition of host peroxidase activity. PLoS Pathog. 8:e1002684. doi: 10.1371/ journal.ppat.1002684

Idnurm, A., Urquhart, A. S., Vummadi, D. R., Chang, S., Van de Wouw, A. P., and López-Ruiz, F. J. (2017). Spontaneous and CRISPR/Cas9-induced mutation of the osmosensor histidine kinase of the canola pathogen Leptosphaeria maculans. Fungal Biol. Biotechnol. 4:12. doi: 10.1186/s40694-017-0043-0

Jiang, W., Zhou, H., Bi, H., Fromm, M., Yang, B., and Weeks, D. P. (2013). Demonstration of CRISPR/Cas9/sgRNA-mediated targeted gene modification in Arabidopsis, tobacco, sorghum and rice. Nucleotide Acids Res. 41, e188. doi: $10.1093 /$ nar/gkt780

Larkan, N. J., Lydiate, D. J., Parkin, I. A. P., Nelson, M. N., Epp, D. J., Cowling, W. A., et al. (2013). The Brassica napus blackleg resistance gene LepR3 encodes a receptor-like protein triggered by the Leptosphaeria maculans effector AVRLM1. New Phytol. 197, 595-605. doi: 10.1111/nph.12043

Larkan, N. J., Ma, L., and Borhan, M. H. (2015). The Brassica napus receptorlike protein RLM2 is encoded by a second allele of the LepR3/Rlm2 blackleg resistance locus. Plant Biotechnol. J. 13, 983-992. doi: 10.1111/pbi.12341

Li, J., Norville, J. E., Aach, J., McCormack, M., Zhang, D., Bush, J., et al. (2013). Multiplex and homologous recombination-mediated genome editing in Arabidopsis and Nicotiana benthamiana using RNA guide and Cas9. Nat. Biotechnol. 31, 684-686. doi: 10.1038/nbt.2654

Liban, S. H., Cross, D. J., Kutcher, H. R., Peng, G., and Fernando, W. G. D. (2016). Race structure and frequency of avirulence genes in the western Canadian Leptosphaeria maculans pathogen population, the causal agent of blackleg in brassica species. Plant Pathol. 65, 1161-1169. doi: 10.1111/ppa.12489

Long, Y., Wang, Z., Sun, Z., Fernando, W. G. D., McVetty, B. E. P., and Li, G. (2011). Identification of two blackleg resistance genes and fine mapping of one of these two genes in a Brassica napus canola variety 'Surpass 400'. Theor. Appl. Genet. 122, 1223-1231. doi: 10.1007/s00122-010-1526-Z

Nødvig, C. S., Nielsen, J. B., Kogle, M. E., and Mortensen, U. H. (2015). A CRISPR-Cas9 system for genetic engineering of filamentous fungi. PLoS One 10:e0133085. doi: 10.1371/journal.pone.0133085

Nováková, M., Šašek, V., Trdá, L., Krutinová, H., Mongin, T., Valentová, O., et al. (2016). Leptosphaeria maculans effector AvrLm4-7 affects Salicylic Acid (SA) and Ethylene (ET) signalling and hydrogen Peroxide (H2 O2) accumulation in Brassica napus. Mol. Plant Pathol. 17, 818-831. doi: 10.1111/mpp.12332

Parlange, F., Daverdin, G., Fudal, I., Kuhn, M. L., Balesdent, M. H., Blaise, F., et al. (2009). Leptosphaeria maculans avirulence gene AvrLm4-7 confers a dual recognition specificity by the Rlm4 and Rlm7 resistance genes of oilseed rape, and circumvents Rlm4-mediated recognition through a single amino acid change. Mol. Microbiol. 71, 851-863. doi: 10.1111/j.1365-2958.2008.06547.x

Petit-Houdenot, Y., Degrave, A., Meyer, M., Blaise, F., Ollivier, B., Marais, C. L., et al. (2019). A two genes-for-one gene interaction between Leptosphaeria maculans and Brassica napus. New Phytol. 223, 397-411. doi: 10.1111/nph. 15762

Plissonneau, C., Daverdin, G., Ollivier, B., Blaise, F., Degrave, A., Fudal, I., et al. (2016). A game of hide and seek between avirulence genes AvrLm4-7 and AvrLm3 in Leptosphaeria maculans. New Phytol. 209, 1613-1624. doi: 10.1111/ nph.13736
Plissonneau, C., Rouxel, T., Chèvre, A. M., Van de Wouw, A. P., and Balesdent, M. H. (2018). One gene-one name: the AvrLmJ1 avirulence gene of Leptosphaeria maculans is AvrLm5. Mol. Plant Pathol. 19, 1012-1016. doi: $10.1111 /$ mpp. 12574

Rimmer, S. R. (2006). Resistance genes to Leptosphaeria maculans in Brassica napus. Can. J. Plant Pathol. 28, S288-S297. doi: 10.1080/07060660609507386

Schuster, M., Schweizer, G., and Kahmann, R. (2018). Comparative analyses of secreted proteins in plant pathogenic smut fungi and related basidiomyceyes. Fungal Genet. Biol. 112, 21-30. doi: 10.1016/j.fgb.2016.12.003

Shan, Q., Wang, Y., Li, J., Zhang, Y., Chen, K., Liang, Z., et al. (2013). Targeted genome modification of crop plants using a CRISPR-Cas system. Nat. Biotechnol. 31, 686-688. doi: 10.1038/nbt.2650

Takayuki, A., Kennosuke, M., Tohru, Y., Ogawa, T., Ohsato, S., Arie, T., et al. (2015). Tailor-made CRISPR/Cas system for highly efficient targeted gene replacement in the rice blast fungus. Biotechnol. Bioeng. 112, 2543-2549. doi: 10.1002/bit.25662

Tonguc, M., and Griffiths, P. D. (2004). Transfer of powdery mildew resistance from Brassica carinata to Brassica oleracea through embryo rescue. Plant Breed. 123, 587-589. doi: 10.1111/j.1439-0523.2004.00987.x

Van de Wouw, A. P., Lowe, R. G. T., Elliott, C. E., Dubois, D. J., and Howlett, B. J. (2014). An avirulence gene, AvrLmJ1, from the blackleg fungus, Leptosphaeria maculans, confers avirulence to Brassica juncea varieties. Mol. Plant Pathol. 15, 523-530. doi: 10.1111/mpp.12105

Van de Wouw, A. P., Marcroft, S. J., Barbetti, M. J., Hua, L., Salisbury, P. A., Rouxel, T., et al. (2009). Dual control of avirulence in Leptosphaeria maculans towards a Brassica napus cultivar with "sylvestris -derived" resistance suggests involvement of two resistance genes. Plant Pathol. 58, 305-313. doi: 10.1111/j. 1365-3059.2008.01982.x

Williams, P. H., and Delwiche, P. A. (1979). "Screening for resistance to blackleg of crucifers in the seedling stage," in Proceedings of a Eucarpia Conference on a Breeding of Cruciferous Crops, eds N. P. A. van Marreqijk and H. Toxopeus (Wageningen: Foundation for Agricultural Plant Breeding, 164-170.

Xie, K., and Yang, Y. (2013). RNA-guided genome editing in plants using a CRISPR-Cas system. Mol. Plant 6, 1975-1983. doi: 10.1093/mp/sst119

Zhang, X., and Fernando, W. G. D. (2018). Insights into fighting against blackleg disease of Brassica napus in Canada. Crop Pasture Sci. 69, 40-47. doi: 10.1071/ CP16401

Zhang, X., Peng, G., Kutcher, H. R., Balesdent, M. H., Delourme, R., and Fernando, W. G. D. (2016). Breakdown of Rlm3 resistance in the Brassica napusLeptosphaeria maculans pathosystem in western Canada. Eur. J. Plant Pathol. 145, 659-674. doi: 10.1007/s10658-015-0819-0

Zhang, X., Peng, G., Parks, P., Hu, B., Li, Q., Jiang, L., et al. (2017). Identifying seedling and adult plant resistance of Chinese Brassica napus germplasm to Leptosphaeria maculans. Plant Pathol. 66, 752-762. doi: 10.1111/ppa.12626

Conflict of Interest: The authors declare that the research was conducted in the absence of any commercial or financial relationships that could be construed as a potential conflict of interest.

Copyright (c) 2020 Zou, Liu, Selin and Fernando. This is an open-access article distributed under the terms of the Creative Commons Attribution License (CC BY). The use, distribution or reproduction in other forums is permitted, provided the original author(s) and the copyright owner(s) are credited and that the original publication in this journal is cited, in accordance with accepted academic practice. No use, distribution or reproduction is permitted which does not comply with these terms. 